\title{
Evaluación de la inmunogenicidad de una proteína recombinante de Pasteurella multocida para la prevención de la neumonía aguda en alpacas (Vicugna pacos)
}

\author{
Evaluation of the imunogenicity of one recombinant protein for the prevention of \\ acute pneumonia in alpacas (Vicugna pacos)
}

\author{
Gerald Moreno-Morales ${ }^{1}$, Jorge Maximiliano G. ${ }^{1}$, Juan Siuce M. ${ }^{1}$, Ana Chero O. ${ }^{1}$, \\ Guido Medina S. ${ }^{2}$, Luis Luna E. ${ }^{1}$, Raúl Rosadio A. ${ }^{1}$, Lenin Maturrano H. ${ }^{1}$
}

\section{Resumen}

\begin{abstract}
El objetivo del presente estudio fue evaluar la capacidad inmunogénica in vivo de una proteína recombinante de membrana externa de Pasteurella multocida denominada P6-like (rP6-like). El ensayo de campo se realizó en una unidad de producción alpaquera en Puno, Perú, y el procesamiento de las muestras en la Facultad de Medicina Veterinaria de la UNMSM. Se formaron 4 grupos de 5 animales, y se inoculó, según el grupo a) solución salina normal, b) bacterina, c) rP6-like, d) combinación de bacterina + rP6-like. Se tomaron muestras de sangre en el día $0,5,7,9,12$ y 15 pos-inoculación. Se diseñaron pruebas de ELISA indirecto de punto único para la cuantificación relativa de anticuerpos específicos anti rP6-like y para anticuerpos anti-proteína total de $P$. multocida. El grupo inoculado con rP6-like mostró elevación significativa de anticuerpos desde el día 7 $(\mathrm{p}<0.05)$ hasta el día 15 pos-inoculación. Los demás productos biológicos no presentaron elevación significativa durante el periodo del estudio. El estudio permitió evidenciar la capacidad inmunogénica de la rP6-like, lo cual permite continuar con futuros estudios de eficacia.
\end{abstract}

Palabras clave: Pasteurella multocida; vacuna; P6-like; Omp16; alpaca; ELISA indirecta

\footnotetext{
${ }^{1}$ Unidad de Biología y Genética Molecular, Facultad de Medicina Veterinaria, Universidad Nacional Mayor de San Marcos, Lima, Perú

${ }^{2}$ Centro de Investigación y Producción La Raya, Universidad Nacional del Altiplano, Puno, Perú

${ }^{3}$ Email: amaturranoh@unmsm.edu.pe
}

Recibido: 2 de abril de 2019

Aceptado para publicación: 8 de junio de 2021

Publicado: 24 de agosto de 2021

CLos autores. Este artículo es publicado por la Rev Inv Vet Perú de la Facultad de Medicina Veterinaria, Universidad Nacional Mayor de San Marcos. Este es un artículo de acceso abierto, distribuido bajo los términos de la licencia Creative Commons Atribución 4.0 Internacional (CC BY 4.0) [https:// creativecommons.org/licenses/by/4.0/deed.es] que permite el uso, distribución y reproducción en cualquier medio, siempre que la obra original sea debidamente citada de su fuente original 
The aim of this study was to evaluate the in vivo immunogenic capacity of a recombinant outer membrane protein of Pasteurella multocida called P6-like (rP6-like). The field trial was carried out in an alpaca production unit in Puno, Peru, and the processing of the samples was done in the Faculty of Veterinary Medicine of San Marcos University in Lima. Four groups of 5 animals were formed, and they were inoculated according to group with a) normal saline solution, b) bacterin, c) rP6-like, d) combination of bacterin + rP6-like. Blood samples were collected on days $0,5,7,9,12$ and 15 post-inoculation. Single-point indirect ELISA tests were designed for the relative quantification of specific anti-rP6-like antibodies and for total anti-total P. multocida protein antibodies. The group inoculated with rP6-like showed significant elevation of antibodies from day $7(\mathrm{p}<0.05)$ until day 15 post-inoculation. The other biological products did not show significant elevation during the studied period. The study showed the immunogenic capacity of rP6like, which allows to continue with future efficacy studies.

Key words: Pasteurella multocida; vaccine; P6-like; Omp16; alpaca; indirect ELISA

\section{INTRODUCCIÓN}

La neumonía aguda de las crías destaca como una de las principales causas de mortalidad en alpacas neonatas y tuis del altiplano en el Perú (FAO, 2005), pudiendo ocasionar mortalidades hasta del 27\% (Ramírez, 1989), lo que se traduce en enormes pérdidas económicas por disminución de individuos logrados al destete, menor proporción de hembras y machos de reemplazo, y reducción de la producción de fibra (FAO, 2005).

Pasteurella multocida (PM) es un cocobacilo Gram negativo causante de graves enfermedades en un gran número de especies animales (Hunt et al., 2000). Estas infecciones son causadas por específicos tipos capsulares (A, B, C y D) y serotipos (116) (Carter, 1952; Heddleston et al., 1972). Además, es el principal agente bacteriano aislado de casos de neumonía aguda en alpacas. Esta bacteria invade el tejido pulmonar luego de un evento de estrés, como exposición al frío extremo, mala nutrición, $\mathrm{o}$ por previa infección viral (Cirilo, 2012; Rosadio et al., 2011). En el Perú ha sido reportado el tipo capsular A y genotipo L6
(Rímac et al., 2017), correspondiente a los serotipos 10, 11, 12 y 15 de PM en alpacas fallecidas por neumonía aguda (Harper et al., 2015).

La pasteurelosis neumónica, nombre que reciben todas las infecciones del parénquima pulmonar provocadas por $P$. multocida es altamente contagiosa y su tratamiento puede ser largo, costoso e inefectivo (Kehrenberg et al., 2001). La prevención mediante medidas de manejo y uso de vacunas son las mejores herramientas para su control (Hurtado et al., 2017). El desarrollo de vacunas implica ensayos in vitro, cuyos resultados deben ser evaluados en la especie de destino para medir su inmunogenicidad y eficacia protectora (OIE, 2008). Diversos autores describen a las proteínas de membrana externa (OMPs) como blancos vacunales promisores en diversas bacterias de importancia en medicina veterinaria, como Bacillus anthracis, Brachispyra hyodisenteriae y Haemophilus parasuis (Ariel et al., 2002; Song et al., 2009; Li et al., 2016). Para la evaluación de la inmunogenicidad de una vacuna usualmente se emplea la prueba de ELISA indirecta, la cual mide el nivel relativo de anticuerpos séricos (Subhash, 2015). 
El uso de herramientas bioinformáticas y el conocimiento del genoma de una cepa de $P$. multocida aislado de un brote de neumonía en alpacas (Hurtado et al., 2017) permitió la determinación in silico de proteínas fuertemente inmunogénicas de PM, dentro de los cuales la proteína P6-like, una OMP asociada al peptidoglicano, tuvo características ideales para su expresión (Juscamayta et al., 2014). Posteriormente, la inmunogenicidad de la rP6-like fue evaluada in vitro, en células mononucleares de sangre periférica (PBMC) de alpaca, donde se corroboró un patrón de expresión celular principalmente de tipo Th2, el cual se relaciona fuertemente con la producción de anticuerpos IgG (Maximiliano et al., 2018). Sin embargo, todavía no se cuenta con estudios que describan el efecto de la rP6-like sobre la inmunidad mediada por anticuerpos en alpacas. El presente estudio tuvo como objetivo determinar la capacidad inmunogénica in vivo de tipo IgG, que puede generar la rP6-like en alpacas.

\section{Materiales y Métodos}

\section{Lugar de Ejecución}

El ensayo de campo se ejecutó en enero de 2017, en el Centro de Investigación y Producción C.I.P. La Raya, de la Universidad del Altiplano. El centro se encuentra en el distrito de Santa Rosa, provincia de Melgar, región Puno, Perú, a 4200 msnm. El procesamiento de las muestras se hizo en la Unidad de Biología y Genética Molecular de la Facultad de Medicina Veterinaria (FMV) de la Universidad Nacional Mayor de San Marcos (UNMSM), Lima, Perú.

\section{Animales y Material Biológico}

Se formaron cuatro grupos de 20 alpacas tuis Huacaya hembras, seleccionadas al azar dentro de un rebaño de aproximadamente 300 tuis hembra. Los animales tenían aproximadamente 12 meses de edad y se encontraban clínicamente sanos. De cada grupo se tomaron cinco animales al azar para el seguimiento. Se inoculó una dosis única de $2 \mathrm{ml}$ a cada animal de cada grupo: $\mathrm{G}_{1}(\mathrm{n}=5) 2 \times 10^{8} \mathrm{UFC} /$ animal de la bacterina; $\mathrm{G}_{2}(\mathrm{n}=5) 10 \mu \mathrm{g} / \mathrm{ani}$ mal de la proteína recombinante $\mathrm{rP6}-\mathrm{like} ; \mathrm{G}_{3}$ $(\mathrm{n}=5) 10 \mu \mathrm{g}$ de rP6-like $+1 \times 10^{8} \mathrm{UFC} /$ animal de ambos productos; $\mathrm{G}_{4}(\mathrm{n}=5$, grupo control) Solución salina normal. La inoculación se realizó por vía subcutánea, en la región axilar derecha.

Se tomaron muestras de sangre de la vena yugular los días 0 (tiempo control), 5, 7, 9,12 y 15 . Se centrifugó la sangre a $1600 \mathrm{~g}$ durante $10 \mathrm{~min}$. Las muestras de suero recuperadas se mantuvieron a $-20{ }^{\circ} \mathrm{C}$ hasta su procesamiento en el laboratorio.

\section{Preparación de la bacterina}

Se utilizó el protocolo empleado por Hsuan et al. (2009). La bacterina está compuesta por una suspensión en Caldo BHI (Brain-Heart Infusion) de P. multocida (cepa nativa), aislada de un caso en alpacas de la región de Puno, a una concentración de $1 \mathrm{x}$ $10^{8} \mathrm{UFC} / \mathrm{ml}$. Fue inactivada con formaldehido al $0.25 \%$ y como adyuvante fue añadido hidróxido de aluminio al $0.25 \%(\mathrm{v} / \mathrm{v})$. La bacterina se almacenó a $4{ }^{\circ} \mathrm{C}$ y se mantuvo con geles refrigerantes durante el procedimiento de inoculación.

Preparación de la proteína recombinante para inoculación

Se utilizó una vacuna de tipo subunidad compuesta por la proteína denominada rP6like obtenida en la Unidad de Biología y Genética Molecular (FMV-UNMSM). Luego del procedimiento de purificación, la proteína fue diluida en PBS y mezclada con hidróxido de aluminio. La mezcla se almacenó a $4{ }^{\circ} \mathrm{C}$ y se mantuvo con geles refrigerantes durante el procedimiento de inoculación. 
Preparación de antígeno total de PM para iELISA

Los antígenos para el inmunoensayo fueron preparados usando el protocolo modificado de Ledesma et al. (2016). Para ello, la cepa de $P$. multocida (cepa nativa) fue cultivada en caldo BHI a $37^{\circ} \mathrm{C}$ por $24 \mathrm{~h}$, con agitación continua. El medio de cultivo fue centrifugado en tubos Falcon de $50 \mathrm{ml}$ a 7000 $\mathrm{g}$ por 15 min y el pellet fue lavado tres veces con buffer fosfato salino PBS. La lisis de las bacterias fue obtenida suspendiendo el pellet en $10 \mathrm{ml}$ de PBS frío y se realizaron tres ciclos de congelación y descongelación en nitrógeno líquido. Se sonicaron las células bacterianas en siete pulsos de $10 \mathrm{~s}$ cada uno, $\mathrm{y}$ fueron centrifugadas a $12000 \mathrm{~g}$ por $30 \mathrm{~min}$ a $4{ }^{\circ} \mathrm{C}$. El sobrenadante fue separado para su uso posterior. La proteína total de PM fue cuantificada mediante fluorometría usando Qubit ${ }^{\mathrm{TM}}$ Thermo Scientific y el lisado fue distribuido en alícuotas y almacenado a $-4{ }^{\circ} \mathrm{C}$ hasta su uso en iELISA.

\section{Preparación de proteína recombinante para ELISA}

Los procedimientos de clonación, expresión y purificación son los mismos que se detallan en «Preparación de la proteína recombinante para inoculación». El producto de esta purificación se cuantificó mediante Qubit $^{\mathrm{TM}}$ Thermo Scientific para diluirla con PBS 0.1M y obtener una dilución final de 100 $\mu \mathrm{g} / \mathrm{ml}$. Esta se almacenó en refrigeración a $4{ }^{\circ} \mathrm{C}$ hasta su evaluación mediante la prueba de iELISA.

\section{Estandarización de iELISAs}

En la estandarización de la primera prueba de ELISA para detección de anticuerpos anti-rP6-like, los pocillos se tapizaron con rP6-like a concentraciones de 100, $50,25,12.5,6.25$ y $3.1 \mu \mathrm{g} / \mathrm{ml}$ y el suero se diluyó a concentraciones de 1/50,1/100,1/200, 1/400,1/800, encontrándose concentraciones óptimas de rP6-like de $3.5 \mu \mathrm{g} / \mathrm{ml}$ y en el sue- ro de $1 / 200$. Para la estandarización de la segunda prueba de ELISA con la proteína total de $P$. multocida se usaron las concentraciones de antígeno de 140, 70, 35, 16.5, 8.25 y $4.1 \mu \mathrm{g} / \mathrm{ml}$ y el suero se diluyó a concentraciones de 1:50, 1:100, 1:200, 1:400 y 1:800, encontrándose concentraciones óptimas de proteína total de $35 \mu \mathrm{g} / \mathrm{ml}$ y en el suero de 1:200. Luego de obtener las concentraciones óptimas de antígeno y suero se probaron concentraciones de agente bloqueante (BSA) de 1,3 y $5 \%(\mathrm{~m} / \mathrm{v})$, donde se eligió la concentración de $3 \%$. El conjugado y cromógeno fueron usados a las concentraciones recomendadas por los fabricantes.

\section{Materiales para iELISA}

Se preparó buffer fosfato salino (PBS) a concentración de $0.1 \mathrm{M}$, el cual sirvió para dilución de todos los reactivos. El buffer de lavado (PBS-T) consistió en una solución de Tween $20 \mathrm{KPL}^{\circledR}$ diluida en PBS a concentración de $0.05 \%$. El suero problema se diluyó a razón de 1:200 en buffer de dilución, el cual está compuesto de albúmina sérica bovina (BSA) al 3\% en PBS 0.1M. El anticuerpo conjugado fue un anticuerpo secundario policlonal de cabra anti-IgG de llama enlazado a la peroxidasa de rábano picante (HRP) Abcam ${ }^{\circledR}$ a concentración final de 1/20 000 . E1 sustrato cromógeno 3, 3', 5, 5'tetramethylbenzidina (TMB) Sureblue ${ }^{\mathrm{TM}}$ se usó a la concentración recomendada por el fabricante (listo para usar). Para todos los procedimientos se usaron placas de poliestireno de alta afinidad de 96 pocillos Corning ${ }^{\circledR}$.

\section{iELISAs de punto único}

Las muestras de suero fueron analizadas por la prueba de ELISA indirecta (iELISA) de punto único siguiendo los protocolos descritos por Ledesma et al. (2016) y Shivachandra et al. (2017) para tapizado con proteína total de PM y rP6-like, respectivamente. Se utilizaron dos tipos de antígenos para recubrir las placas de poliestireno. La 
placa de ELISA fue tapizada con la proteína P6-like diluida en buffer fosfato salino $0.1 \mathrm{M}$ (PBS), pH 7.4, a una concentración de 3.5 $\mu \mathrm{g} / \mathrm{ml}$ para la evaluación de inmunidad contra la proteína rP6-like. Para la evaluación de la inmunidad contra la bacterina y contra rP6-like + bacterina, se tapizó la placa con $100 \mu 1$ de proteína total de PM a una concentración de $35 \mu \mathrm{g} / \mathrm{ml}$ mediante incubación a $37^{\circ} \mathrm{C}$ durante $1 \mathrm{~h}$. Posteriormente se hicieron tres lavados con $200 \mu 1$ de PBS-T. Luego se bloquearon los pocillos con $200 \mu \mathrm{l}$ de BSA $3 \%$ a $4{ }^{\circ} \mathrm{C}$ durante $16 \mathrm{~h}$. Seguidamente se efectuaron tres lavados con PBS-T y se añadió por duplicado, $100 \mu 1 /$ pocillo de los sueros problema a dilución de 1/200 en solución de dilución, incubándose a $37{ }^{\circ} \mathrm{C}$ por $1 \mathrm{~h}$. Se volvieron a realizar tres lavados para luego añadir $100 \mu \mathrm{ldel}$ anticuerpo conjugado con HRP a dilución 1/20 000. Se incubó una vez más a $37^{\circ} \mathrm{C}$ por $1 \mathrm{~h}$, se realizaron tres lavados más y se añadió $100 \mu 1$ sustrato cromógeno (TMB). Finalmente se incubó a temperatura ambiente durante $10 \mathrm{~min}$ en la oscuridad. La placa fue leída a una longitud de onda de $650 \mathrm{~nm}$ en un espectrofotómetro multicanal Biorad $\AA$.

\section{Análisis Estadístico}

Se compararon las medianas de $\mathrm{OD}_{650}$ del día cero y demás días dentro de los grupos $G_{1}, G_{3}$ y $G_{4}$ mediante la prueba de Wilcoxon. Se hizo un segundo análisis comparando las medianas de los $\mathrm{OD}_{650}$ del $\mathrm{G}_{4} \mathrm{y}$ del $G_{2}$ en cada día de seguimiento mediante la prueba U de Mann-Whitney con un nivel de significancia de 0.05 . El software para el análisis estadístico y los gráficos fueron STATA ${ }^{\circledR} 15.0$ y GraphPadPrism $7{ }^{\circledR}$, respectivamente.

\section{Consideraciones Éticas}

El manejo de los animales se realizó causando el menor estrés posible. Los muestreos tuvieron una duración promedio de
$2 \mathrm{~h}$ por día, desde las 07:00 hasta las 09:00 para no interrumpir el horario de alimentación de los animales. El protocolo de toma de muestra de sangre se realizó tomando en cuenta el método publicado por Morton et al. (1993).

\section{Resultados}

La inoculación de la bacterina (en $\mathrm{G}_{1}$ ) produjo un pico de elevación de anticuerpos significativamente superior al del día cero $(\mathrm{p}<0.05)$; sin embargo, los niveles de IgG en los días 12 y 15 pos-inoculación no fueron significativamente diferentes al día cero ( $\mathrm{Fi}$ gura 1, Cuadro 1). En el Cuadro 2 se observa que $4 / 5$ de animales del $\mathrm{G}_{1}$ aumentaron los niveles de IgG en el día 15 en comparación con sus niveles basales (día 0 ).

En el grupo inoculado con P6-like $\left(\mathrm{G}_{2}\right)$, desde el séptimo día en adelante se evidenció una elevación significativa de los niveles de IgG con respecto al grupo control $(p<0.05)$, siendo el pico máximo de IgG el día 15 (Figura 2, Cuadro 1). En el Cuadro 3 se visualiza que $5 / 5$ animales del $\mathrm{G}_{2}$ elevaron los niveles de $\operatorname{IgG}$ en el día 15 con respecto al grupo control.

En el $\mathrm{G}_{3}$, la inoculación de rP6-like + bacterina no estimuló un aumento significativo en los días de seguimiento con respecto al día cero (p>0.05) (Figura 3, Cuadro 1). En el Cuadro 4 se observa que $3 / 5$ animales disminuyeron sus niveles de $\operatorname{IgG}$ en el séptimo día, y $5 / 5$ animales disminuyeron sus niveles de IgG en el día 15 con respecto al día cero.

El grupo control no mostró variaciones significativas en los niveles de IgG durante los periodos de evaluación. Además, la desviación estándar fue pequeña en cada día de seguimiento ( $\mathrm{SD} \leq 0.10)$ (Cuadro 1). Individualmente, los niveles de IgG disminuyeron hasta el día 15 (Cuadro 5). 
Cuadro 1. Medidas de tendencia central y de dispersión de los cuatro grupos experimentales: $\left(\mathrm{G}_{1}\right)$ inoculado con bacterina, $\left(\mathrm{G}_{2}\right)$ inoculado con proteína rP6-like, $\left(\mathrm{G}_{3}\right)$ inoculado con bacterina + rP6-like, $\left(\mathrm{G}_{4}\right)$ control

\begin{tabular}{cccccccccccccc}
\hline \multirow{2}{*}{ Día } & \multicolumn{3}{c}{ Grupo 1 } & \multicolumn{4}{c}{ Grupo 2 } & \multicolumn{3}{c}{ Grupo 3 } & \multicolumn{3}{c}{ Grupo 4 } \\
\cline { 2 - 12 } & Media $^{1}$ & DE & EE & Media $^{2}$ & DE & EE & Media $^{1}$ & DE & EE & Media $^{1}$ & DE & EE \\
\hline 0 & $0.440^{\mathrm{a}}$ & 0.097 & 0.043 & $0.572^{\mathrm{a}}$ & 0.187 & 0.840 & $0.575^{\mathrm{a}}$ & 0.182 & 0.082 & $0.480^{\mathrm{a}}$ & 0.097 & 0.043 \\
5 & $0.401^{\mathrm{a}}$ & 0.031 & 0.037 & $0.528^{\mathrm{a}}$ & 0.148 & 0.066 & $0.500^{\mathrm{a}}$ & 0.130 & 0.058 & $0.430^{\mathrm{a}}$ & 0.093 & 0.042 \\
7 & $0.441^{\mathrm{a}}$ & 0.111 & 0.050 & $0.847^{\mathrm{b}}$ & 0.121 & 0.054 & $0.443^{\mathrm{a}}$ & 0.067 & 0.030 & $0.446^{\mathrm{a}}$ & 0.083 & 0.037 \\
9 & $0.581^{\mathrm{b}}$ & 0.060 & 0.027 & $0.894^{\mathrm{c}}$ & 0.241 & 0.130 & $0.553^{\mathrm{a}}$ & 0.063 & 0.028 & $0.412^{\mathrm{a}}$ & 0.100 & 0.045 \\
12 & $0.554^{\mathrm{a}}$ & 0.133 & 0.060 & $1.090^{\mathrm{d}}$ & 0.549 & 0.160 & $0.565^{\mathrm{a}}$ & 0.070 & 0.031 & $0.412^{\mathrm{a}}$ & 0.064 & 0.028 \\
15 & $0.577^{\mathrm{a}}$ & 0.088 & 0.040 & $1.092^{\mathrm{e}}$ & 0.281 & 0.169 & $0.609^{\mathrm{a}}$ & 0.052 & 0.023 & $0.433^{\mathrm{a}}$ & 0.081 & 0.037 \\
\hline
\end{tabular}

1 Promedios en la misma columna con letras diferentes son estadísticamente diferentes con respecto al día 0 de su mismo grupo $(p<0.05)$

2 Promedios en la columna del grupo inoculado con la rP6-like $\left(G_{2}\right)$ con letras diferentes son significativamente diferentes con el grupo control $\left(\mathrm{G}_{4}\right)$

DE: Desviación estándar; EE: Error estándar

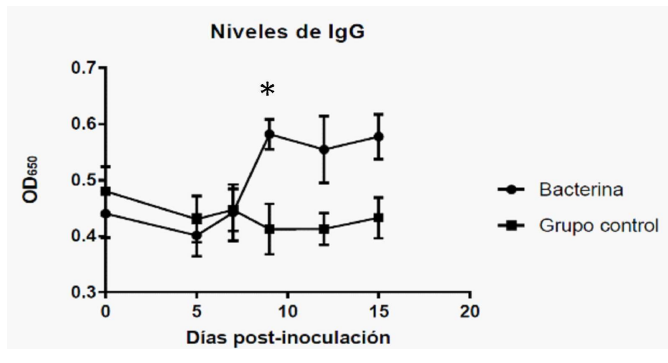

Figura 1. Resultados del ELISA para los niveles promedio de $\operatorname{IgG}\left(\mathrm{OD}_{650}\right)$ anti-proteína total de Pasteurella mulcocida en el grupo inoculado con una bacteria $\left(\mathrm{G}_{1}\right)$. Las barras de error son los errores estándar. *: Valor diferente al día cero $(\mathrm{p}<0.05)$

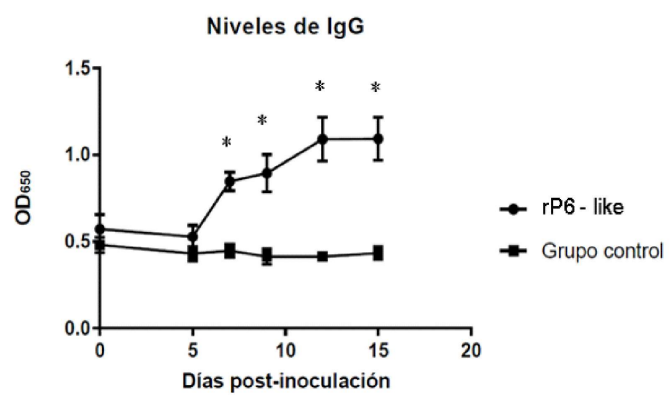

Figura 2. Resultados del ELISA para los niveles de IgG (OD650) anti- rP6-like en el grupo inoculado con P6-like $\left(\mathrm{G}_{2}\right)$. Las barras de error son los errores estándar. *: Valor diferente al día cero del grupo control $(\mathrm{p}<0.05)$
La zona de inoculación de no presentó alteraciones inflamatorias ni signos de enfermedad en ninguno de los animales.

\section{Discusión}

El producto biológico que generó la mayor cantidad de anticuerpos con relación al día cero fue la rP6-like. Estos resultados complementan los hallazgos de Maximiliano et al. (2018), quienes encontraron que la rP6like estimula una respuesta de tipo $\mathrm{Th} 2$ (IgG). Sumado a esto, los resultados concuerdan con Sivachandra et al. (2017), quiénes obtuvieron una elevación significativa de la IgG en el día 21 en ratones. No obstante, la elevación de IgG no fue tan marcada como los llevados a cabo en estudios en ratones (Okay et al., 2012; Shivachandra et al., 2014) debido, probablemente, a que las alpacas ya estaban expuestas a la proteína P6-like. Los resultados de la proteína son más alentadores a corto plazo que los demás productos biológicos, probablemente, debido a que la naturaleza proteica de la P6-like produjo un mayor estímulo sobre las células CD4+ Th2, con la consecuente formación de centros 
Cuadro 2. Densidades ópticas para los niveles de $\operatorname{IgG}\left(\mathrm{OD}_{650}\right)$ anti-proteína total de Pasteurella multocida en cada animal inoculados con la bacterina $\left(\mathrm{G}_{1}\right)$

\begin{tabular}{ccccccc}
\hline \multirow{2}{*}{ Animal } & \multicolumn{7}{c}{ Días pos-inoculación } \\
\cline { 2 - 7 } & 0 & 5 & 7 & 9 & 12 & 15 \\
\hline 1 & 0.438 & 0.355 & 0.425 & 0.606 & 0.566 & 0.636 \\
2 & 0.501 & 0.442 & 0.507 & 0.541 & 0.492 & 0.494 \\
3 & 0.539 & 0.525 & 0.512 & 0.561 & 0.546 & 0.696 \\
4 & 0.437 & 0.316 & 0.511 & 0.675 & 0.765 & 0.566 \\
5 & 0.285 & 0.367 & 0.252 & 0.526 & 0.403 & 0.495 \\
\hline
\end{tabular}

Cuadro 3. Densidades ópticas para los niveles de $\operatorname{IgG}\left(\mathrm{OD}_{650}\right)$ anti-rP6-like en cada uno de los animales inoculados con la rP6-like $\left(\mathrm{G}_{2}\right)$

\begin{tabular}{ccccccc}
\hline \multirow{2}{*}{ Animal } & \multicolumn{7}{c}{ Días pos-inoculación } \\
\cline { 2 - 7 } & 0 & 5 & 7 & 9 & 12 & 15 \\
\hline 1 & 0.687 & 0.609 & 0.866 & 1.019 & 1.285 & 1.327 \\
2 & 0.581 & 0.435 & 0.639 & 0.665 & 0.747 & 0.812 \\
3 & 0.803 & 0.748 & 0.940 & 1.050 & 1.284 & 1.416 \\
4 & 0.470 & 0.465 & 0.924 & 0.603 & 0.819 & 0.825 \\
5 & 0.320 & 0.384 & 0.865 & 1.132 & 1.316 & 1.081 \\
\hline
\end{tabular}

Cuadro 4. Densidades ópticas para los niveles de $\operatorname{IgG}\left(\mathrm{OD}_{650}\right)$ anti-proteína total de Pasteurella multocida en cada animal inoculado con la bacterina + rP6like $\left(\mathrm{G}_{3}\right)$

\begin{tabular}{ccccccc}
\hline \multirow{2}{*}{ Animal } & \multicolumn{7}{c}{ Días pos-inoculación } \\
\cline { 2 - 7 } & 0 & 5 & 7 & 9 & 12 & 15 \\
\hline 1 & 0.865 & 0.634 & 0.354 & 0.620 & 0.656 & 0.638 \\
2 & 0.401 & 0.354 & 0.479 & 0.541 & 0.504 & 0.533 \\
3 & 0.620 & 0.479 & 0.506 & 0.461 & 0.486 & 0.583 \\
4 & 0.539 & 0.398 & 0.388 & 0.539 & 0.598 & 0.625 \\
5 & 0.450 & 0.634 & 0.490 & 0.605 & 0.583 & 0.666 \\
\hline
\end{tabular}


Cuadro 5. Densidades ópticas para los niveles de $\operatorname{IgG}\left(\mathrm{OD}_{650}\right)$ anti rP6-like en cada animal inoculado con solución salina normal $\left(\mathrm{G}_{4}\right)$

\begin{tabular}{ccccccc}
\hline \multirow{2}{*}{ Animal } & \multicolumn{7}{c}{ Días pos-inoculación } \\
\cline { 2 - 7 } & 0 & 5 & 7 & 9 & 12 & 15 \\
\hline 1 & 0.546 & 0.415 & 0.479 & 0.456 & 0.433 & 0.487 \\
2 & 0.467 & 0.446 & 0.477 & 0.377 & 0.410 & 0.396 \\
3 & 0.428 & 0.450 & 0.366 & 0.346 & 0.389 & 0.425 \\
4 & 0.356 & 0.290 & 0.357 & 0.317 & 0.327 & 0.322 \\
5 & 0.603 & 0.549 & 0.554 & 0.566 & 0.503 & 0.534 \\
\hline
\end{tabular}

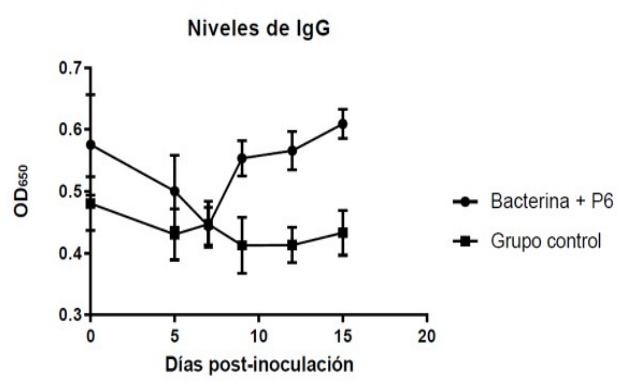

Figura 3. Resultados del ELISA para los niveles promedio de $\operatorname{IgG}\left(\mathrm{OD}_{650}\right)$ anti-proteína total de Pasteurella multocida en el grupo inoculado con rP6-like + bacterina $\left(\mathrm{G}_{3}\right)$. Las barras de error son los errores estándar

germinativos y generación de células B de memoria, ya que ha sido una exposición secundaria al antígeno, lo cual es compatible con lo formulado por Vinuesa et al. (2005) y Siegrist (2008).

La bacterina produjo una elevación de anticuerpos a partir del noveno día, pero decayeron al día 15. Estos resultados son compatibles con los hallazgos de Sun (2009), quién reportó una pobre inmunogenicidad estimulada por una bacterina de $P$. multocida inoculada en ovejas. Esta ineficacia de las bacterinas también se ha observado en estudios con Mycoplasma hyopneumoniae (Fisch et al., 2016), Actinobacillus pleuropneu- moniae (Shao et al., 2010) y Streptococcus suis (Hsueh et al., 2017). Este suceso podría deberse a la composición múltiple de la bacterina que conlleva a una pobre estimulación de los centros germinativos (Siegrist, 2008). Esto también ha podido ocurrir debido a la disminución en la concentración de las proteínas P6-like expresadas por la cepa de $P$. multocida por la «domesticación» de la cepa laboratorial (Fisch et al., 2016).

Los dos productos combinados no resultaron en una elevación de los niveles de anticuerpos, a diferencia del trabajo de Hsuan et al. (2009), quiénes comprobaron que los anticuerpos aumentan considerablemente al inmunizar cerdos con rPMT junto con una bacterina. El resultado del presente estudio pudo deberse a que la bacterina posee un efecto deletéreo o anérgico, tal como los expuestos en el estudio de Dabo et al. (2007). Si este fuera el caso, la bacterina junto con la rP6-like podría ser una mala combinación para continuar con ensayos de validación.

El grupo control presentó ligeras desviaciones con respecto a la media del día 0 (Figuras 1-3), posiblemente debido a factores no controlados, como la temperatura ambiental, la cual es un factor influyente sobre la reactividad de los monocitos (Arias et al., 2016). El tiempo control de todos los grupos (día 0 ) presentó también niveles muy altos de 
anticuerpos a diferencia de otros estudios (Okay et al., 2012; Shivachandra et al., 2014; Wei et al., 2017) debido a que, probablemente, $P$. multocida presente en la microbiota estimuló la producción de anticuerpos anti rP6-like y anti-proteína total de P. multocida (De Steenhuijsen et al., 2015). Esto representa una limitante de las pruebas serológicas en agentes que forman parte de la microbiota; sin embargo eso no ha sido un impedimento para comprobar la eficacia vacunal, tal es el caso de la vacuna contra la enterotoxemia en alpacas (Rosadio et al., 2012).

Adicionalmente se comprobó que la inoculación de los tres productos biológicos no produce efectos adversos visibles en animales jóvenes, lo cual contribuye a los estudios de inocuidad. Estos resultados son compatibles con los trabajos de Moreno et al. (2016) y de Shivachandra et al. (2017) en alpacas y ratones, respectivamente.

Dentro de las limitaciones de este estudio, los resultados de densidades ópticas de los tres productos biológicos no fueron comparables, debido a las dos pruebas de iELISA que se utilizaron para la medición de IgG (Crowther et al., 2009). Así mismo, el tamaño de muestra reducido utilizado en el ensayo limitó el poder estadístico; sin embargo, las diferencias encontradas entre $\mathrm{G}_{1}$ y el grupo control fueron suficientes para no atribuirlas al error aleatorio (Triola et al., 2009). Por otro lado, el uso exclusivo de hembras tuis limita la generalización de los resultados, pese a que aún no existe evidencia que la inmunidad humoral sea diferente entre alpacas macho y hembra, a diferencia de ovinos (Sun, 2009).

Estos primeros ensayos con la proteína recombinante han permitido conocer su capacidad inmunogénica, pues se ha logrado establecer que rP6-like es capaz de generar anticuerpos específicos de manera significativa y la respuesta generada en estos primeros ensayos indican que, de manera prelimi- nar, podría actuar mejor sola que en combinación con una bacterina. Serán necesarios más estudios para conocer el nivel mínimo protector de anticuerpos y la eficacia de la vacuna elegida.

\section{Conclusión}

La rP6-like estimula la producción de anticuerpos IgG en alpacas tui hembras.

\section{Agradecimientos}

Los autores agradecen al Programa Nacional de Innovación para la Competitividad y Productividad INNÓVATE Perú (FINCyT), Proyecto Contrato N. ${ }^{\circ}$ 133FINCyT-IB-2013 «Vacunología reversa: Desarrollo de una vacuna de nueva generación para el control y/o prevención de la neumonía pasteurelósica en alpacas», y al Programa de Promoción de Tesis de Pregrado del Vicerrectorado de Investigación y Postgrado - UNMSM, 2017, 2 ${ }^{\text {da }}$ Etapa, por su apoyo en el financiamiento del presente trabajo. También, se agradece a todo el equipo del CIP «La Raya» UNA, por el apoyo decisivo en la logística y muestreo de campo, así como a Dennis Carhuaricra Huamán, Raquel Quino Quispe y Carla Durán Gonzales por los consejos recibidos y el apoyo sustancial en el análisis de muestras en el laboratorio.

\section{Literatura Citada}

1. Arias N, Velapatinoa B, Hunga A, Cokb J. 2016. Cytokines expression in alpacas and llamas exposed to cold stress. Small Ruminant Res 141: 135-140. doi: 10.1016/j.smallrumres.2016.-07.016

2. Ariel N, Zvi A, Grosfeld H, Gat O, Inbar Y, Velan B, Cohen S, Shafferman $A$. 2002. Search for potential vaccine candidate open reading frames in the Bacillus anthracis virulence plasmid pXO1: in silico and in vitro screening. Infect Immun 70: 6817-6827. doi: 10.1128/IAI.70.12.6817-6827.2002 
3. Carter GR. 1952. The type specific capsular antigen of Pasteurella multocida. Can J Med Sci 30: 48-53. doi: 10.1139/cjms52-008

4. Cirilo CE, Manchego SA, Rivera HH, Rosadio AR. 2012. Coexistencia de virus y bacterias en Neumonías agudas en alpacas neonatas. Rev Inv Vet Perú 23: 317-335. doi: 10.15381/rivep.v23i3.914

5. Crowther JR. 2009. Methods in molecular biology: the ELISA guidebook. $2^{\text {nd }}$ ed. Austria: Humana Press. 564 p.

6. De Steenhuijsen PW, Sanders EA, Bogaert D. 2015. The role of the local microbial ecosystem in respiratory health and disease. Philos T Roy Soc B 370: 20140294. doi: 10.1098/rstb.2014.0294

7. Dabo SM, Taylor JD, Confer AW. 2007. Pasteurella multocida and bovine respiratory disease. Anim Health Res Rev 8: 129-50. doi: 10.1017/S1466252307001399

8. [FAO] Organización de las Naciones Unidas para la Alimentación y la Agricultura. 2005. Situación actual de los camélidos sudamericanos en el Perú: Proyecto de cooperación técnica en apoyo a la crianza y aprovechamiento de los camélidos sudamericanos en la región andina. Roma: FAO. [Internet]. Disponible en: https://tarwi.lamolina.edu.pe/ $\sim$ emellisho/zootecnia_archivos/situacion\%20alpcas\%20peru.pdf

9. Fisch A, Marchioro B, Klazer C, Galli $V$, Rodrigues $N$, Simionatto $S$, Odir Dellagostin A, et al. 2016. Commercial bacterins did not induce detectable levels of antibodies in mice against Mycoplasma hyopneumoniae antigens strongly recognized by swine immune system. Trials Vaccinol 5: 32-37. doi: 10.1016/ j.trivac.2016.01.001

10. Harper M, John M, Turni IC, Edmunds M, Michael FST, Adler B, Blackall PJ, et al. 2015. Development of a rapid multiplex PCR to genotype Pasteurella multocida strains using the lipopolysaccharide outer core biosynthesis locus. J Clin Microbiol 53: 477-485. doi: 10.1128/JCM.02824-14
11. Heddleston KL, Gallagher JE, Rebers $P A$. 1972. Fowl cholera: gel diffusion precipitin test for serotyping Pasteurella multocida from avian species. Avian Dis 16: 925-936. doi: 10.2307/1588773

12. Hsuan SL, Liao CM, Huang C, Winton JR, Chen ZW, Lee WC. 2009. Efficacy of a novel Pasteurella multocida vaccine against progressive atrophic rhinitis of swine. Vaccine 27: 2923-2929. doi: 10.1016/j.vaccine.2009.03.005

13. Hsueh K, Cheng L, Lee J, Chung Y, Chung W, Chu C. 2017. Immunization with Streptococcus suis bacterin plus recombinant Sao protein in sows conveys passive immunity to their piglets. BMC Vet Res 13: 1-9. doi: 10.1186/s12917016-0937-8

14. Hunt ML, Adler B, Townsend KM. 2000. The molecular biology of Pasteurella multocida. Vet Microbiol 72: 3-25. doi: 10.1016/j.vaccine.2009.-03.005

15. Hurtado R, Aburjaile F, Mariano D, Canário M, Benevides L, Fernandez D, Allasi N, et al. 2017. Draft genome sequence of a virulent strain of Pasteurella multocida isolated from alpaca. J Genomics 5: 68-70. doi: 10.7150/ jgen. 19297

16. Juscamayta E, Maturrano L, Fernández D, Hurtado R, Allasi N, Rosadio R. 2014. Whole genome analysis of Pasteurella multocida strain UNMSM to identify potential vaccine candidates against acute pneumonia. In: SCB-Latin American meeting on bioinformatics with BSB. Sao Paulo.

17. Kehrenberg C, Tanzil GS, Martel JL, Dancla EC, Schwarz S. 2001. Antimicrobial resistance in Pasteurella and Mannheimia: epidemiology and genetic basis. Vet Res 32: 323-339. doi: 10.1051/vetres:2001128

18. Ledesma MM, Díaz AM, Barberis C, Vay C, Manghi MA, Leoni J, Castro MS, Ferrari A. 2016. Identification of Lama glama as reservoirs for Acinetobacter lwoffii. Front Microbiol 8: 278. doi: 10.3389/fmicb.2017.00278 
19. Li M, Li C, Song S, Kang H, Yang D, Li G 2016. Development and antigenic characterization of three recombinant proteins with potential for Glässer's disease prevention. Vaccine 34: 22512258. doi: 10.1016/j.vaccine.2016.03.014

20. Maximiliano J, Maturrano L, Luna L, Hurtado R, Chero A, Rosadio $R$. 2018. Evaluación de la inmunogenicidad de una proteína recombinante de una Pasteurella multocida aislada de alpacas con neumonía. Rev Inv Vet Perú 29:339-348. doi: 10.15381/rivep.v29i1.14197

21. Moreno-Morales G, Maximiliano GJ, Medina SG, Rosadio AR, Maturrano HL. 2016. Evaluación de la inocuidad de una bacterina de Pasteurella multocida para la prevención de neumonía aguda en crías de alpaca. En: XXII Congreso Nacional de Ciencias Veterinarias. Huánuco, Perú.

22. Morton DB, Abbot D, Barclay R, Close BS, Ewbank R, Gask D, Heath M, et al. 1993. Removal of blood from laboratory mammals and birds. Laboratory Animals 27: 1-22.

23. [OIE] Organización Mundial de Sanidad Animal. 2008. Principios de producción de vacunas veterinarias. Manual de la OIE sobre animales terrestres. 17 p. [Internet]. Disponible en: https:// www.oie.int/fileadmin/Home/esp/ Health_standards/tahm/1.01.08_Principios_produccion_vacunas_veterin.pdf

24. Okay S, Özcengiz E, Gürsel I, $\ddot{O}_{z c e n g i z} G$ 2012. Immunogenicity and protective efficacy of the recombinant Pasteurella lipoprotein E and outer membrane protein $\mathrm{H}$ from Pasteurella multocida A: 3 in mice. Res Vet Sci 93: 1261-1265. doi: 10.1016/j.rvsc.2012.05.011

25. Ramírez A. 1989. Enfermedades infecciosas en camélidos sudamericanos. En: XII Reunión Científica Anual APPA. Lima: Asociación Peruana de Producción Animal.
26. Rímac R, Luna L, Hurtado R, Rosadio R, Maturrano L. 2017. Detection and genetic characterization of Pasteurella multocida from alpaca (Vicugna pacos) pneumonia cases. Trop Anim Health Pro 49: 1325-1328. doi: 10.1007/ s11250-017-1309-5

27. Rosadio R, Cirilo E, Manchego A, Rivera $H .2011$. Respiratory syncytial and Parainfluenza type 3 viruses coexisting with Pasteurella multocida and Mannheimia hemolytica in acute pneumonias of neonatal alpacas. Small Ruminant Res 97: 110-116. doi: 10.1016/ j.smallrumres.2011.02.001

28. Siegrist C. 2008. Correlates of vaccineinduced immunity. In: Plotkin SA, Orenstein WA, Offit PA (eds). $5^{\text {th }}$ ed. Philadelphia: Saunders/Elsevier. p 17-36.

29. Shao M, Wang Y, Wang C, Yang Guo, Peng Y, Liu J, Li G. 2010. Evaluation of multicomponent recombinant vaccines against Actinobacillus pleuropneumoniae in mice. Acta Vet Scand 52: 18. doi: 10.1186/1751-0147-52-52

30. Shivachandra SB, Kumar A, Yogisharadhya $R$. 2014. Immunogenicity of highly conserved recombinant VacJ outer membrane lipoprotein of Pasteurella multocida. Vaccine 32: 290-296. doi: 10.1016/j.vaccine.2013.10.075

31. Shivachandra SB, Kumar A, Mohanty NN and Yogisharadhya R. 2017. Immunogenicity of recombinant Omp 16 protein of Pasteurella multocida B: 2 in mouse model. Indian J Anim Sci 87: 29-34.

32. Song Y, La T, Phillips ND, Bellgard MI, Hampson DJ. 2009. A reverse vaccinology approach to swine dysentery vaccine development. Vet Microbiol 137: 111-119. doi: 10.1016/j.vetmic.2008.12.018

33. Sun T. 2009. Evaluation of a vaccine of vaccine against Mannheimia haemolytica and Pasteurella multocida in sheep. PhD Thesis. USA: Cornell University. $47 \mathrm{p}$. 
34. Subhash K. 2015. Development of ELISA techniques for haemorrhagic septicaemia. Int J Bioassays 4: 457-4577.

35. Triola M. 2009. Estadística. $10^{\circ}$ ed. México. Pearson. $904 \mathrm{p}$.

36. Vinuesa CG, Tangye $S C$, Moser B. 2005. Follicular B helper $\mathrm{T}$ cells in antibody responses autoimmunity. Nat
Rev Immunol 5: 853-865. doi: 10.1038/ nri1714

37. Wei X, Wang Y, Luo R, Qian W, Sizhu $S$, Zhou H. 2017. Identification and characterization of a protective antigen, PlpB of bovine Pasteurella multocida strain LZ-PM. Dev Comp Immunol 71: 1-7. doi: 10.1016/j.dci.2017.01.017 